Artigo

\title{
Uma Análise da Aplicação de Três Métodos Estatísticos para o Cálculo do Desvio Padrão da Direção do Vento na Região Tropical
}

\author{
Adaiana Francisca Gomes da Silva ${ }^{1}$, Edson Luiz Zaparoli ${ }^{1}$, Gilberto Fisch ${ }^{2}$ \\ ${ }^{1}$ Divisão de Engenharia Mecânica Aeronáutica, Departamento de Turbomáquinas, \\ Instituto Tecnológico de Aeronáutica, São José dos Campos, SP, Brasil. \\ ${ }^{2}$ Divisão de Ciências Atmosféricas, Instituto de Aeronáutica e Espaço, \\ São José dos Campos, SP, Brasil.
}

Recebido 27/2/2014 - Aceito 30/4/2015

\begin{abstract}
Resumo
Neste trabalho é apresentada uma revisão sobre métodos para cálculo do desvio padrão da direção do vento horizontal $\left(\sigma_{\theta}\right)$. Três diferentes métodos clássicos encontrados na literatura [Verral e Williams, 1982 (VW), Ackermann, 1983 (ACK), e Yamartino, 1984 (YM)] são avaliados a partir de intercomparação entre eles utilizando-se dados de vento obtidos por radiossondagens. Estas medidas foram realizadas a cada seis horas durante um período de 11 dias do mês de setembro de 2008 (estação seca da região Norte do NE do Brasil, com ventos alísios intensos). Os cálculos mostraram que o método de YM apresentou-se o mais confiável, sendo que os resultados de VW foram muito próximos de YM. Comparando os métodos dois a dois, as máximas diferenças foram de 29 graus entre ACK e YM e 26,3 graus entre ACK e VW, enquanto que entre YM e VW a máxima diferença foi de apenas 2,6 graus. Segundo a literatura revisada, YM é o método mais estável ao longo da variação de $\sigma_{\theta}$, com erro de, no máximo, $2 \%$.
\end{abstract}

Palavras chave: desvio padrão, perfil vertical, radiossondagem, ventos alísios.

\section{An Analysis of Applying Three Statistical Methods for Calculating the Standard Deviation of the Wind Direction at the Tropical Region}

\begin{abstract}
In this paper, a review about three classical methods for calculating the standard deviation of the horizontal wind direction $\left(\sigma_{\theta}\right)$ is presented. These methods described by Verral and Williams, $1982(\mathrm{VW})$, Ackermann, 1983 (ACK), and Yamartino, 1984 (YM) are evaluated by intercomparison between them using wind data obtained from radiosonde measurements. These measurements were taken every six hours over a period of 11 days of September 2008 (dry season of the north of NE region of Brazil, with strong trade winds). Calculations indicated that YM method showed to be the most reliable, whereas the results of VW were very close to YM. Comparing the methods two by two, the maximum differences were 29 degrees between ACK and YM and 26.3 degrees between ACK and VW, while between YM and VW the maximum difference was only 2.6 degrees. According to the reviewed literature, YM is the most stable method over the range of $\sigma_{\theta}$, with maximum error of $2 \%$.
\end{abstract}

Keywords: standard deviation, vertical profile, radiosondes, trade winds.

\section{Introdução}

O vento, ou o movimento do ar, é um fenômeno natural e extremamente variável, tanto em sua velocidade quanto em sua direção, passando, inclusive, por momentos de velocidade nula. Mudanças abruptas e repentinas na dire- ção do vento podem causar diversos problemas. Dentre eles, um exemplo são as turbinas eólicas, que têm sua eficiência diminuída, além do agravamento do fenômeno de fadiga mecânica nas pás (Mahrt, 2011).

Estas variações, que dependem de diversos fatores como a radiação solar, latitude do local, complexidade do 
terreno, interações mecânicas, correntes de convecção e força de Coriolis (Farrugia e Micallef, 2006), refletem na interpretação de conjuntos de dados que tenham sido coletados ao longo de um determinado espaço ou de um período temporal. Em muitos casos, um agrupamento de dados, por si só, não apresenta um comportamento padrão ou regular, não fornecendo um resultado relevante. Entretanto, métodos estatísticos podem ser utilizados para comparar diferentes conjuntos e, então, obter resultados interessantes destes dados.

Calcular quantidades estatísticas da direção do vento, tal como seu desvio padrão, tem sido um problema de longa data, pois esta é uma variável periódica e circular com descontinuidade no limite $0-360^{\circ}$. Por isto, métodos estatísticos padrões comumente aplicáveis a conjuntos de dados lineares não devem ser utilizados, requerendo um tratamento especial. Para resolver este problema, diversos algoritmos já foram propostos para o cálculo da média e/ou do desvio padrão de variáveis circulares, tais como aqueles descritos em Verrall e Williams (1982), Ackermann (1983) e Yamartino (1984), citados, por exemplo, em Turner (1986), Weber (1997), Farrugia e Micallef (2006, 2009), Namboodiri et al. (2014), entre outros. Apesar de se tratarem de publicações antigas, estes trabalhos devem ser valorizados como clássicos do tratamento matemático do vento. Outros estimadores são também encontrados, como os propostos por Castans e Barquero (1994) e Ibarra (1995), citados em Farrugia e Micallef (2006), porém geram indeterminações algébricas em algumas condições e estas limitações matemáticas os levam à inviabilidade prática.
Armazenar todos os dados obtidos durante o período de medições para realizar os cálculos posteriormente exige muita memória e capacidade computacional do sistema de aquisição de dados. Para evitar este custo, pode-se parametrizar o desvio padrão do vento $\left(\sigma_{\theta}\right)$ como uma função de momentos de variáveis lineares (como o seno e cosseno do ângulo de direção), onde totais são acumulados sem armazenar os $n$ valores individuais medidos. Por isto, é de grande interesse obter métodos simples para estimar $\sigma_{\theta}$. Exemplos importantes de aplicabilidade do desvio padrão do vento estão relacionados em problemas de turbulência atmosférica e de transporte e dispersão de materiais devido à difusão inerente ao movimento do ar. (Weber, 1997; Farrugia et al., 2009). Segundo Mahrt (2011), é possível analisar a intensidade da turbulência pela variabilidade da direção do vento.

Portanto, tendo em vista a importância deste parâmetro e os poucos estudos associados, este trabalho visa analisar três diferentes métodos (Verrall e Williams, 1982; Ackermann, 1983, e Yamartino, 1984) comumente utilizados na área da Meteorologia. O objetivo é compará-los, apresentando as equações já em suas formas mais simplificadas, além de outras fórmulas importantes usadas no tratamento matemático do vento.

\section{Metodologia}

O vento é uma quantidade tridimensional que, ao ser decomposta, apresenta as componentes horizontais U (zonal) e V (meridional) e vertical (W). Este trabalho considerará apenas as componentes $\mathrm{U}$ e $\mathrm{V}$, ou seja, o vento horizontal.

Tabela 1 - Sequência de cálculos para estimativa do desvio-padrão da direção do vento $\left(\sigma_{\theta}\right)$ segundo o método de Verral e Williams (1982).

\begin{tabular}{|c|c|c|c|}
\hline$S_{a}=\frac{\sum_{i=1}^{n} \operatorname{sen}\left(\theta_{i}\right)}{n}$ & (1) & $N_{2}=\left|C_{a}\right|-\sigma_{c}$ & (7) \\
\hline$C_{a}=\frac{\sum_{i=1}^{n} \cos \left(\theta_{i}\right)}{n}$ & (2) & $N_{1}=\left|C_{a}\right|+\sigma_{c}$ & (8) \\
\hline$\sigma_{S}=\left[\frac{\sum_{i=1}^{n} \operatorname{sen}^{2}\left(\theta_{i}\right)}{n}-S_{a}^{2}\right.$ & (3) & $M_{1}=\sqrt{\left(E_{1}^{2}+N_{1}^{2}\right)}$ & (9) \\
\hline$\sigma_{C}=\left\lceil\frac{\sum_{i=1}^{n} \cos ^{2}\left(\theta_{i}\right)}{n}-C_{a}^{2}\right.$ & (4) & $M_{2}=\sqrt{\left(E_{2}^{2}+N_{2}^{2}\right)}$ & (10) \\
\hline$E_{1}=\left|S_{a}\right|-\sigma_{S}$ & (5) & $\sigma_{\theta}=\frac{1}{2} \cos ^{-1}\left(\frac{E_{1} E_{2}+N_{1} N_{2}}{M_{1} M_{2}}\right)$ & (11) \\
\hline$E_{2}=\left|S_{a}\right|+\sigma_{S}$ & (6) & & \\
\hline
\end{tabular}

sendo $n$ o número de medidas a serem submetidas à análise estatística e $\theta_{i}$ os valores individuais de direção, em radianos. Consequentemente, $\sigma_{\theta}$ também é obtido em radianos. $S_{a}$ e $C_{a}$ são parâmetros de média e $\sigma$ representa variâncias das observações em termos de senos e cossenos. 
Tabela 2 - Sequência de cálculos para estimativa do desvio-padrão da direção do vento $\left(\sigma_{\theta}\right)$ segundo o método de Ackermann (1983).

$\sigma_{U}^{2}=\frac{\sum_{i=1}^{n} U_{i}^{2}}{n}-\bar{U}^{2}$
$\sigma_{V}^{2}=\frac{\sum_{i=1}^{n} V_{i}^{2}}{n}-\bar{V}^{2}$
$\sigma_{\theta}=\left[\left(\bar{V}^{2} \sigma_{U}^{2}+\bar{U}^{2} \sigma_{V}^{2}-2 \bar{U} \bar{V} \sigma_{U V}\right)^{1 / 2}\right] \overline{V e l}^{-2}$
onde $U_{i}$ e $V_{i}$ são valores individuais de velocidade horizontal decomposta, em $\mathrm{m} / \mathrm{s}$; a denotação "barra" significa a média da variável e $\overline{V e l}$ a magnitude do
vento médio, definida conforme a Eq. (16).

\subsection{Descrição dos métodos}

\subsubsection{Verral e Williams}

Verral e Williams (1982) desenvolveram um método de cálculo (daqui em diante referenciado como VW) assumindo que os ângulos sejam normalmente distribuídos e seus cálculos partem da média dos senos e cossenos da direção do vento, e não dos ângulos propriamente, como mostrados na Tabela 1 .

\subsubsection{Ackermann}

Na sequência, em 1983, G. R. Ackermann (daqui em diante, ACK) publicou uma nova metodologia prometendo cálculos mais compactos e economia computacional. Aqui, as variáveis são apresentadas em termos das componentes do vento horizontal e não em termos dos ângulos, como mostrados na Tabela 2.

$$
\overline{V e l}=\left(\bar{U}^{2}+\bar{V}^{2}\right)^{1 / 2}
$$

\subsubsection{Yamartino}

Yamartino (1984) propôs um novo método (YM), testado para distribuições unimodais (Tabela 3).

\subsection{Dados de vento}

Para realizar a intercomparação entre os três métodos, foram utilizados os dados de vento obtidos por radiossondagem durante a campanha Murici II, realizada pelo Instituto de Aeronáutica e Espaço (IAE) no Centro de Lançamento de Alcântara (CLA) durante o período de 16 a 26/09/2008, representativo da estação seca da região e sendo caracterizado por ventos fortes. Maiores informa-

Tabela 3 - Cálculos para estimativa do desvio-padrão da direção do vento $\left(\sigma_{\theta}\right)$ segundo o método de Yamartino (1984).

$$
\sigma_{\theta}=\operatorname{sen}^{-1}(\varepsilon)\left[1+\left(\frac{2}{\sqrt{3}}-1\right) \varepsilon^{3}\right]
$$

$$
\varepsilon=\sqrt{1-\left(S_{a}^{2}+C_{a}^{2}\right)}
$$

onde $S_{a}$ e $C_{a}$ são calculados conforme Eqs. (1) e (2), ou seja, da mesma forma como propõe VW. ções sobre estes dados podem ser obtidas em Silva e Fisch (2014). Durante este período, foram realizadas quatro sondagens diárias, às 00:00, 06:00, 12:00 e 18:00 UTC. Embora a resolução vertical original destas sondagens seja alta, não passando de 15 metros o intervalo entre duas medidas, os níveis nos quais os instrumentos realizam as leituras não são padronizados em todas as radiossondagens. Por este motivo, a fim de possibilitar uma metodologia de trabalho com várias observações em conjunto, os níveis foram padronizados através da interpolação linear dos dados de 50 em 50 metros até $5000 \mathrm{~m}$ de altitude. Para cada nível, foi obtido um valor médio de 11 dias, separado por horário, cujo cálculo foi baseado na publicação de Teixeira e Girardi (1978), conforme Eq. (19):

$$
\bar{\theta}=\cos ^{-1}\left(-\frac{\bar{V}}{\overline{V e l}}\right)
$$

Os resultados dos cálculos de $\sigma_{\theta}$ pelos três diferentes métodos serão apresentados em forma de perfis verticais (Figura 1), onde a linha central representa o valor médio e as linhas laterais pontilhadas representam os desvios padrão inferior (esquerda) e superior (direita), respectivamente.

\section{Resultados e Discussão}

Os perfis verticais gerados a partir do cálculo das três metodologias (Figura 1) apresentaram características semelhantes para os quatro horários. Comparando os três métodos, YM geralmente concorda muito bem com VW, mas ACK apresenta regiões de comportamento diferente dos outros dois. Estas diferenças são percebidas principalmente ao redor das altitudes de 1000 e de $4500 \mathrm{~m}$, aproximadamente. Em uma análise quantitativa, a máxima discrepância encontrada foi de $29^{\circ}$ entre os valores de $\sigma_{\theta}$ estimados por dois métodos diferentes (neste caso, entre YM e ACK, aos $1200 \mathrm{~m}$ de altitude, no horário das 06:00 UTC, sob valores de $\sigma_{\theta} \leq 42^{\circ}$ ). Porém, estes valores altos foram casos pontuais e podem ser relacionados a particularidades atmosféricas já que, na média, as diferenças ficaram abaixo dos $4^{\circ}$. A Figura 2 mostra a diferença (em graus) entre os valores estimados pelos três métodos, com- 

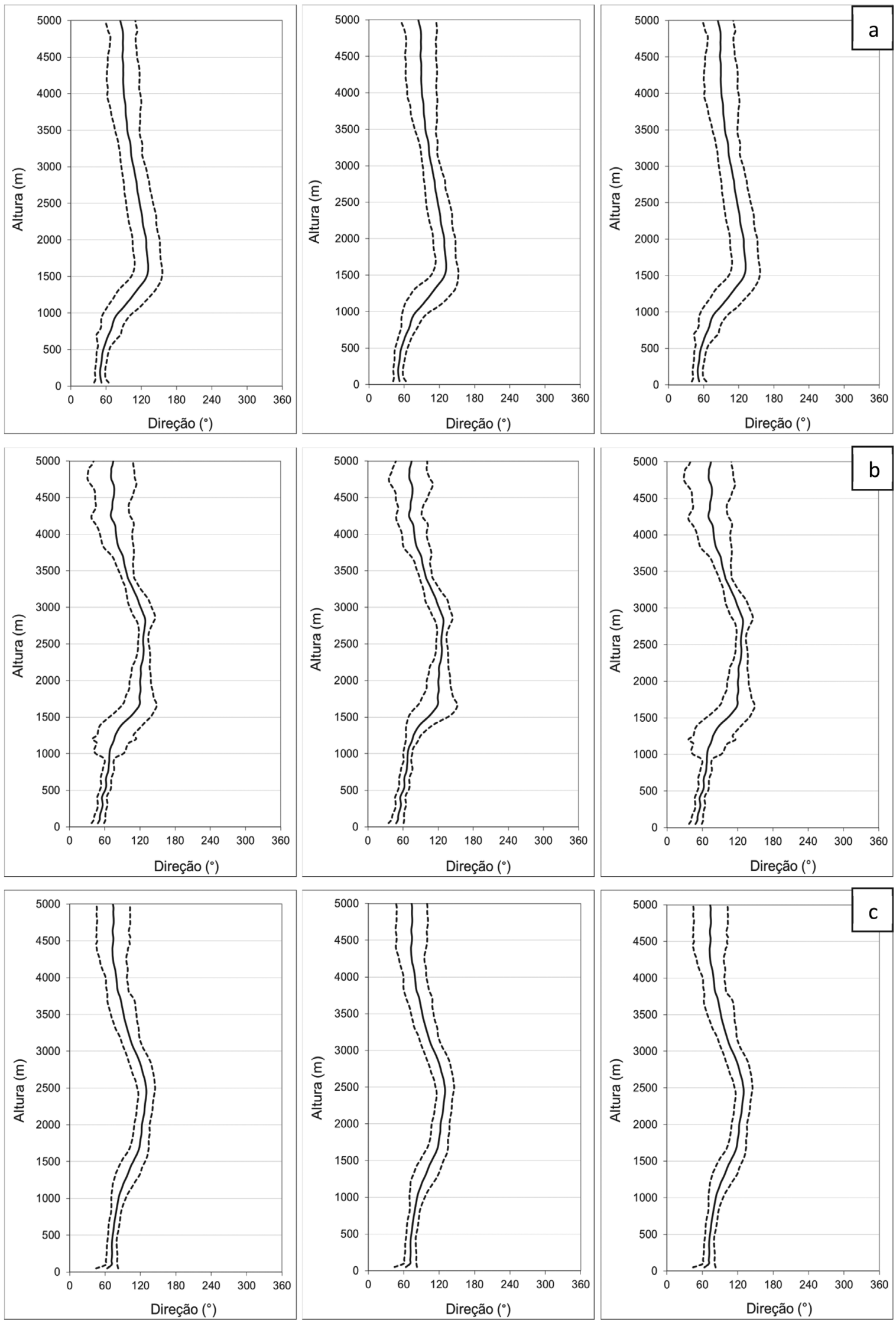

Figura 1 - Variação vertical do desvio padrão da direção do vento pelos métodos VW (esquerda), ACK (central) e YM (direita) para as 00:00 (a), 06:00 (b), 12:00 (c) e 18:00 UTC (d). Perfis obtidos para o período de 16 a 26/09/2008. 

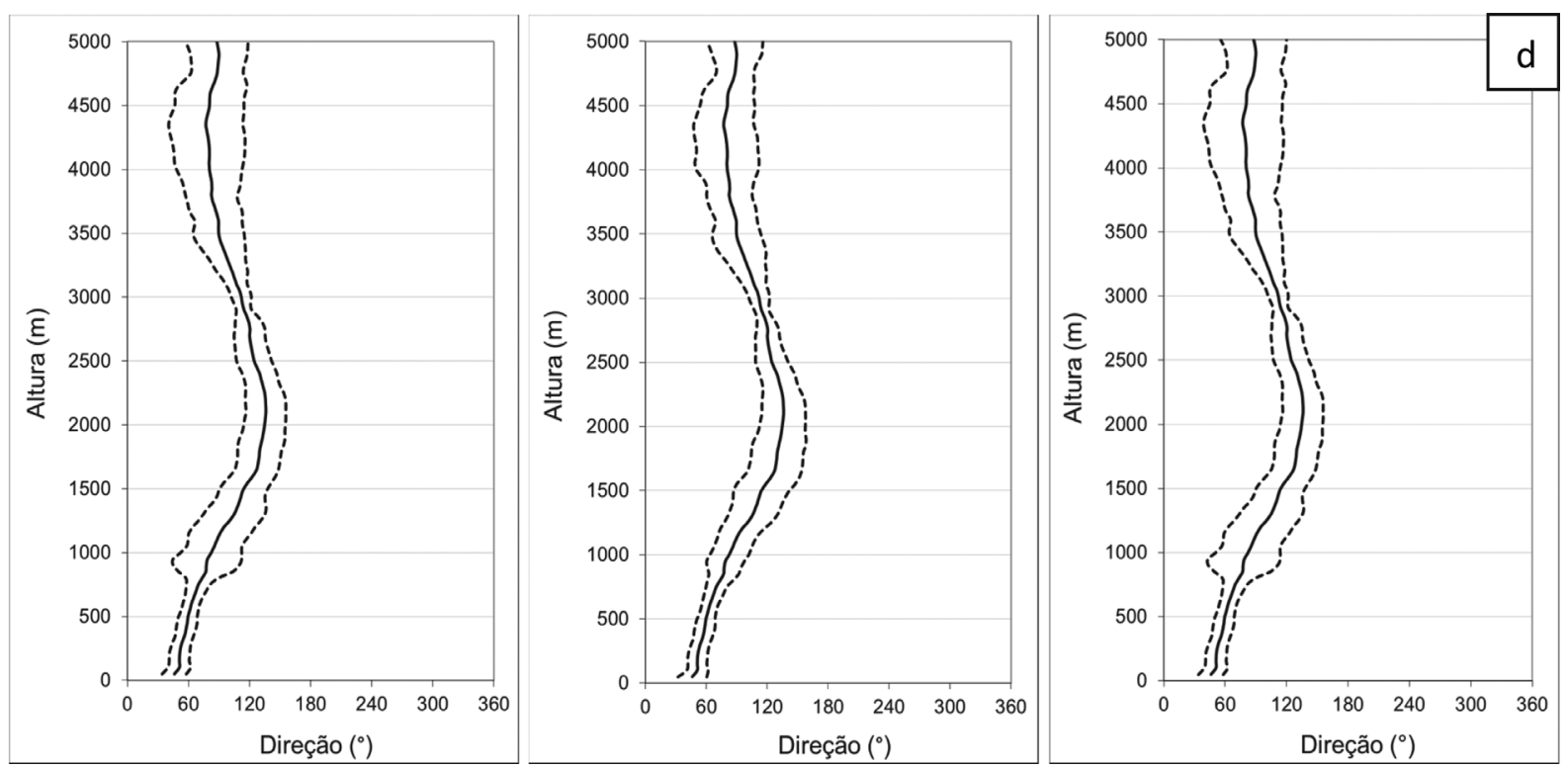

Figura 1(cont.) -

parados dois a dois, apresentados para diferentes níveis de altitude. Pode-se observar que, dentre as três linhas de cada gráfico, os valores mais baixos são sempre as diferenças entre YM e VW, o que, conforme já dito, havia sido percebido qualitativamente a partir dos perfis da Figura 1. Estas diferenças entre YM e VW são pequenas comparadas às maiores diferenças que, na maioria das vezes, estão entre YM e ACK.

Segundo Turner (1986), valores baixos de velocidade e fortes rajadas de vento, causados por passagem de sistemas atmosféricos e/ou presença de brisa marítima/terrestre, assim como turbulência mecânica causada por irregularidades do terreno, contribuem para a elevação do desvio padrão. Namboodiri et al. (2014) também verificaram que, se na região atuam escoamentos de mesoescala e de escala sinótica em direções opostas, o desvio-padrão resultante é elevado. A característica geográfica da região do CLA, onde os dados foram coletados, reforçam estas hipóteses. Além de sofrer influência da Zona de Convergência Intertropical, de brisa marítima e dos ventos alísios, é uma região costeira com mudança abrupta da rugosidade do terreno, sendo possível que um método estatístico seja mais sensível a estas variações do que outro. Além disto, em suas conclusões, Yamartino (1984) ainda reforça que sua fórmula foi validada apenas para distribuição unimodal das direções do vento e que, sob condições de grandes oscilações do vento, o método pode gerar problemas de "aliasing", apesar de que estes aparecerão somente para valores altos de $\sigma_{\theta}$.

Yamartino (1984) e Turner (1986) notaram que o método de ACK superestima o desvio padrão (2 a 3 vezes) quando este possui valores elevados $\left(\sigma_{\theta} \sim 90^{\circ}\right)$, enquanto VW o subestima (erro de $13,5 \%$ para $\sigma_{\theta} \sim 101^{\circ}$ ). Os próprios autores Verral e Williams (1982) reconhecem este problema. A Tabela 7 de Turner (1986) apresenta um estudo detalhado sobre o desempenho de VW, ACK e YM em termos de erros absolutos e percentuais.

Turner (1986) repetiu os resultados de Yamartino (1984), confirmando que o método deste apresenta o menor erro $( \pm 2 \%)$ na intercomparação com outros trabalhos. Este erro foi estimado em comparação com a definição tradicional de desvio padrão acrescida de considerações para variáveis circulares, que foi chamada de método de dois-passos. Os três métodos aqui tratados são considerados de passo único. Isto está relacionado com o número de vezes em que é necessário acessar a memória de armazenamento do sistema de aquisição de dados que está fazendo a conversão estatística. (Yamartino, 1984; Weber, 1997). O presente trabalho enfatizou a comparação entre-métodos e somente diferenças absolutas, em graus, foram calculadas. Não foi utilizado um parâmetro base para que erros percentuais fossem estimados.

Ainda segundo Turner (1986), em termos de erros, o método de YM mostra-se mais estável ao longo de toda a amplitude da variação dos desvios padrão encontrados, enquanto os outros dois métodos apresentaram erros crescentes com o aumento do desvio padrão. Este comportamento falho foi novamente reconhecido por Verral e Williams (1982), mas estes afirmam que o viés é pequeno o suficiente para ser negligenciado.

Segundo Farrugia e Micallef (2006), o método de ACK é capaz de estimar o desvio padrão da direção do vento corretamente apenas enquanto a dispersão angular é baixa e, no geral, apresenta um índice de erros alto e inaceitável, levando os autores a descartar sua utilização. Todavia, no caso do presente trabalho, como os ventos na região são fortes (sempre superiores a 5,0 m/s), a amplitude da direção (dispersão angular) do vento é pequena. Farrugia 

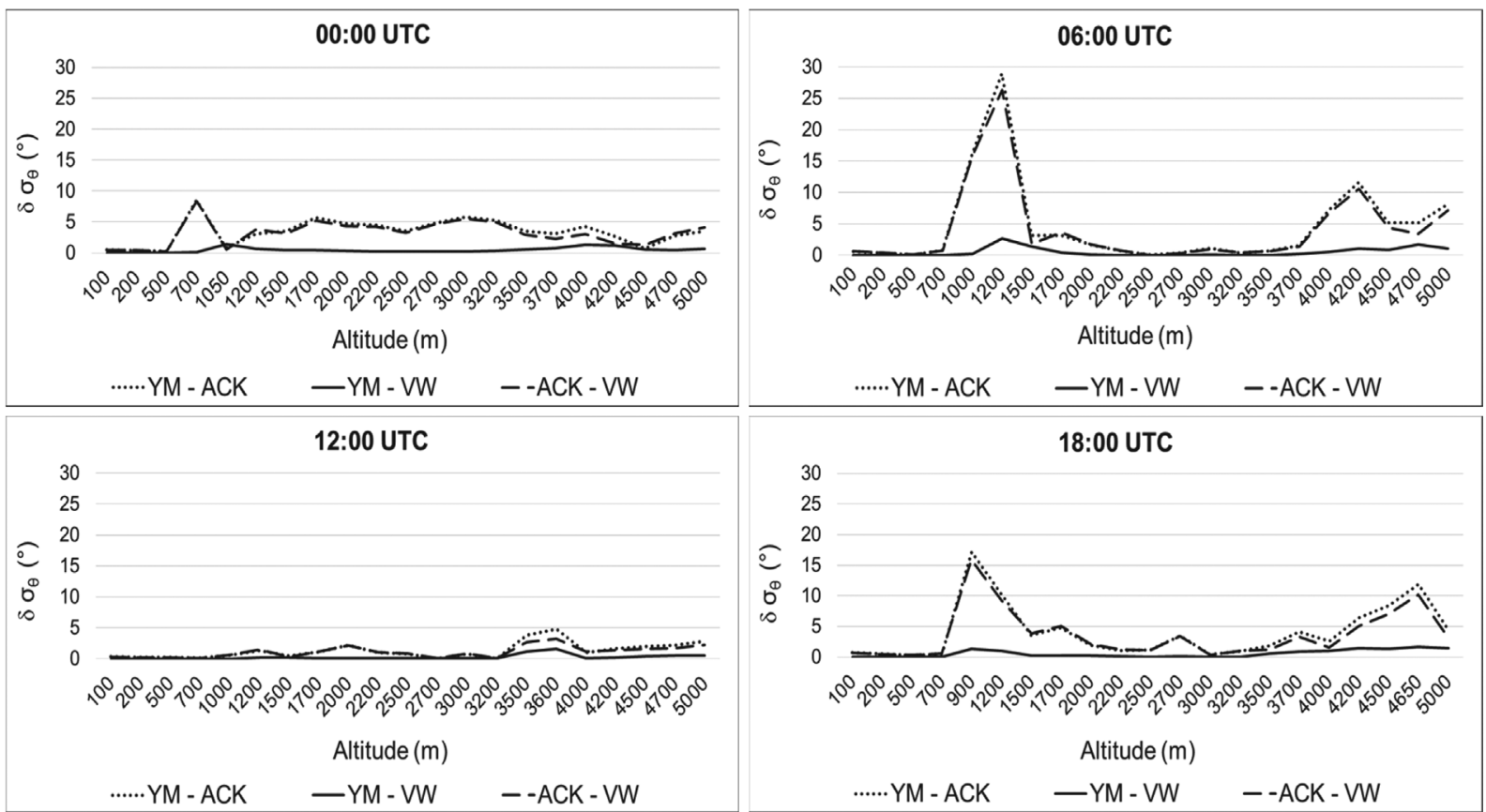

Figura 2 - Diferenças entre os desvios padrão $\left(\delta \sigma_{\theta}\right)$ estimados pelos três métodos, comparados dois a dois em função da altitude para os quatro diferentes horários do período de 16 a 26/09/2008.

e Micallef (2006) também encontraram uma leve superestimação nos cálculos de YM, embora tenha sido considerado o método mais adequado.

\section{Conclusões}

Em todos os testes realizados e de acordo com a literatura científica revisada, o método de YM mostrou os melhores resultados, com erros máximos contabilizados (na literatura) em $\pm 2 \%$ em comparação com a definição tradicional multi-passo de desvio padrão. Além disso, é um método mais simples, com poucas equações a serem resolvidas e de pouca exigência computacional. Por outro lado, tanto na literatura quanto nos resultados próprios deste trabalho, o método de ACK apresentou-se o mais discrepante em comparação com os outros dois.

Sendo assim, recomenda-se o uso do método YM para os cálculos de $\sigma_{\theta}$.

\section{Agradecimentos}

Ao Instituto de Aeronáutica e Espaço (IAE) pelo fornecimento dos dados de vento utilizados na realização deste estudo, através do Prof. Gilberto Fisch.

\section{Referências}

ACKERMANN, G. R. Means and standard deviations of horizontal wind components. Journal of Climate and Applied Meteorology, v. 22, n. 5, p. 959-961, 1983.
CASTANS, M.; BARQUERO, C. G. A framework for the structure of a low wind speed field. Boundary Layer Meteorology, v. 69, p. 137 - 147, 1994.

FARRUGIA, P. S.; MICALLEF, A. Comparative analysis of estimators for wind direction standard deviation. Meteorological Applications, v. 13, p. 29-41, 2006.

FARRUGIA, P. S.; BORG, J. L.; MICALLEF, A. On the algorithms used to compute the standard deviation of wind direction. Journal of Applied Meteorology and Climatology, v. 48, p. 2144-2151, 2009.

IBARRA, J. I. A new approach for the determination of horizontal wind direction fluctuations. Journal of Climate and Applied Meteorology, v. 34, p. 1942-1949, 1995.

MAHRT, L. Surface wind direction variability. Journal of Applied Meteorology and Climatology, v. 50, p. 144-152, 2011.

NAMBOODIRI, K. V. S.; et al. Coastal boundary layer characteristics of wind, turbulence and surface roughness parameter over the Thumba Equatorial Rocket Launching Station, India. Journal of Climatology, v. 2014, p. 1-21, 2014.

SILVA, A. F. G.; FISCH. G. Avaliação do modelo WRF para a previsão do perfil do vento no Centro de Lançamento de Alcântara. Revista Brasileira de Meteorologia, v. 29, n. 2, p. 259-270, 2014.

TEIXEIRA, L.; GIRARDI, C. ECA-04/78: Oscilações do vento da estratosfera equatorial, Adendo B: decomposição do vento. ACA/IAE. São José dos Campos, SP, 1978.

TURNER, D. B. Comparison of three methods for calculating the standard deviation of the wind direction. Journal of Climate and Applied Meteorology, v. 25, n. 5, p. 703-707, 1986. 
VERRALL, K. A.; WILLIAMS, R. L. A method for estimating the standard deviation of wind directions. Journal of Applied Meteorology, v. 21, n. 12, p. 1922-1925, 1982.

WEBER, R. O. Estimators for the standard deviation of horizontal wind direction. Journal of Applied Meteorology, v. 36, n. 10, p. 1403-1415, 1997.
YAMARTINO, R. J. A comparison of several "single-pass" estimators of the standard deviation of wind direction. Journal of Climate and Applied Meteorology, v. 23, n. 9, p. 13621366, 1984.

All the contents of this journal, except where otherwise noted, is licensed under a Creative Commons Attribution License CC-BY. 